\title{
Applications of Aptamers in Medicine: A Mini Review
}

\author{
Nusrat Nahar ${ }^{1}$, Ridwan Bin Rashid ${ }^{1}$ and Mohammad Sharifur Rahman ${ }^{2}$ \\ ${ }^{1}$ Computational Chemistry and Bioinformatics Laboratory, Department of Pharmacy, State University of \\ Bangladesh, Dhaka- 1205, Bangladesh \\ ${ }^{2}$ Department of Pharmaceutical Chemistry, University of Dhaka, Dhaka-1000, Bangladesh
}

Received: January 10, 2017; Accepted: January 26, 2017; Published (Web): March 19, 2017

\begin{abstract}
Aptamers are single-stranded DNA or RNA molecules that bind to its target with high affinity and specificity. Nucleic acid aptamers are an attractive class of carrier molecules because of their tissue penetration capability, high chemical flexibility, low immunogenicity, low toxicity and cost effective production. These characteristics make aptamers an alternative to the antibody. An aptamer can be developed for the treatment of age-related macular degeneracy disease, blood clotting, cancer and auto-immune diseases etc. Macugen is so far the only aptamer-based drug that received FDA approval. Cancer patients are treated by targeting two proteins named nucleolin and CXCL12. Targeted delivery of aptamers has been successfully developed that reduce the occurrence of the unwanted off-target effects. Aptamers are susceptible to renal filtration and endonuclease cleavage. Conjugation with PEG or cholesterol and chemical modification can decrease renal clearance and increase serum stability.
\end{abstract}

Key words: Aptamer, SELEX, Macugen, Coagulation process, Cancer, Autoantibody

\section{Introduction}

Aptamers are synthetic single strand oligonucleotides (ssDNA or RNA) that bind to multiple targets such as proteins, toxins. Aptamers are regarded as an alternative to antibodies because of their high affinity and specificity for target molecules. Since aptamers are chemically synthesized, they can be used for different diagnostic and therapeutic purposes by introducing chemical modification. Aptamers are smaller in size and are able to penetrate into biological compartment more efficiently. They exhibit low immunogenicity (Pendergrast et al., 2005) and good thermal stability. Because of these characteristics, aptamer is considered as an effective probe for the recognition of ligands. Pharmacokinetic properties of aptamers are hard to predict. Aptamers are evolved by a process named SELEX (Systematic Evolution of Ligands by Exponential Enrichment). The library of single stranded DNA molecule is prepared from double stranded PCR products and a RNA library is prepared from transcription of double stranded DNA molecule.
Macugen, a pegylated anti-vascular growth factor aptamer, was the first granted FDA approved drug used against macular degeneracy disease (Sundaram et al., 2013).

SELEX and aptamer: Aptamers are generated by SELEX process. The first step of the cell-SELEX process is the incubation of synthesized oligonucleotide with the target molecules (Figure 1). Then bound oligonucleotides are eluted and amplified to prepare the enriched oligonucleotide pool. This oligonucleotide pool can also be counter-selected by incubating the pool with unwanted targets in order to diminish non-specific binders. Counter selection thereby enhances the specificity of the enriched pool. To obtain high affinity aptamer, multiple rounds of target selection and enrichment can be done. Finally cloning and sequencing of the last PCR product is done to obtain the individual aptamer sequences. Further optimization and modification can produce high affinity aptamer that can be used for different applications.

Correspondence to: Mohammad Sharifur Rahman, E-mail: msr@du.ac.nd 
Oligonucleotide library

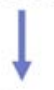

Incubation of the library with the targets

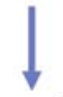

Elution of bound oligonucleotide from the target

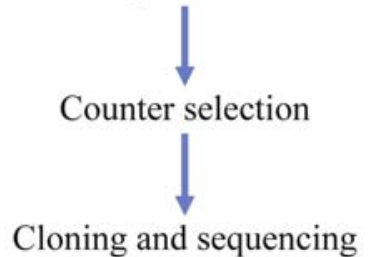

Figure 1. SELEX process.

\section{Therapeutic application of aptamer:}

Aptamers for the treatment of age related macular degeneration (AMD): AMD is the result of deterioration of the macula, a vital part of the retina and causes blindness in a patient if untreated. Vascular endothelial growth factor (VEGF) releases a hormone that causes abnormal vessel growth. AMD is caused by this abnormal blood vessel growth. Macugen, brand name of pegaptanib sodium, is the first aptamer-based drug. It is an RNA aptamer that consists of 28 nucleotides. It targets VEGF and binds to all growth factors except the smallest VEGF121. It disrupts the interaction between VEGF and its receptor and prevents blood vessel growth (Figure 2). It also prevents the formation of defective blood vessels. Pegaptanib is given into the vitreous part to the eye. Aptamers can be administered both subcutaneously and intravenously (Vinores et al., 2006). Treatment by this aptamer has some side effects in human such as inflammation, infection and bleeding inside of the eyes. However, in animal models, no immunogenic responses were recorded.

Aptamer Macugen + VEGF

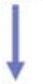

Inhibit interaction between VEGF and its receptor

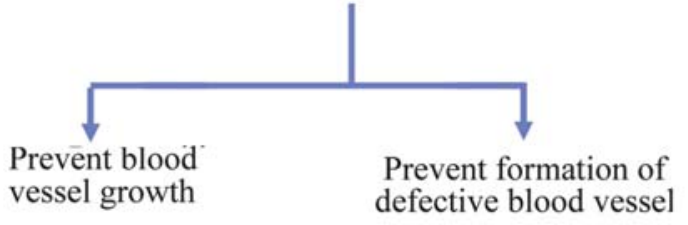

Figure 2. Macugen against AMD.
ARC1905 is a new aptamer-based drug that is effective against AMD. AMD is caused by the hyper action of alternative complement pathway. Chromosomal mutation in the 1q31 region results in defective factor $\mathrm{H}$, which activates complement protein C5. A cascade reaction starts forming membrane attack complex (MAC) that kills retinal cells (Parashar et al., 2016). ARC1905 binds to complement component C5 and stops this cascade reaction (Figure 3). A $40 \mathrm{kDa}$ polyethylene glycol (PEG) is conjugated at the $5^{\prime}$ position of ARC1905 and capped at the 3'-terminus with an inverted nucleotide (Keefe et al., 2010) to increase the half-life.

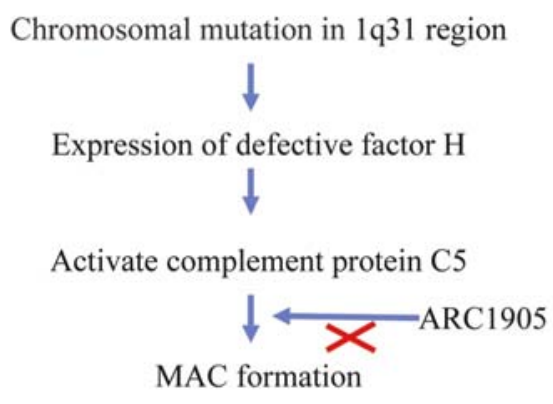

Figure 3. ARC1905 for AMD treatment.

Aptamer in coagulation process: Last step of the blood coagulation process is the formation of thrombin. HD1 or ARC183 is a $15 \mathrm{bp}$ single stranded DNA aptamer. It forms a stable G-quadruplex. By binding to pro-exosite I on both prothrombin and thrombin (Block et al., 1992; Kretz et al., 2006; Kretz et al., 2010), it inhibits pro-exocite I function. This aptamer inhibits the interaction of fibrinogen with thrombin and also competes with FVa for prothrombin binding (Figure 4). By binding with pro-exocite I, HD1 also inhibits platelet PAR interactions and inhibits thrombinmediated platelet aggregation and activation (Griffin et al., 1993). Several anticoagulation activities justify the use of HD1 in cardiac operation. HD1 has a short halflife and hence for active anticoagulation activity, a continuous infusion is required.

$\mathrm{Nu} 172$ is another 26 nucleotide DNA aptamer. It was developed by Archemix/Nuvelo and is more potent than ARC183. It is now in phase II clinical trial. HD22 is another DNA aptamer and is 29 nucleotides long. It inhibits thrombin mediated activation of platelets and FV/FVIII activation (Tasset et al., 1997). HD1 and 
HD22 were joined with a poly-dA linker to form a hybrid aptamer which shows tighter binding and more efficacious anticoagulation activity (Muller et al., 2008).

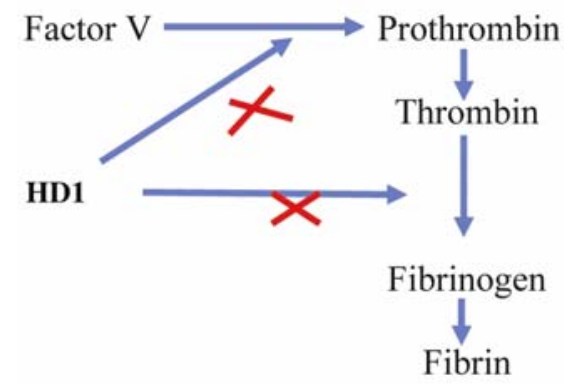

Figure 4. HD1 block fibrinogen and thrombin interaction and compete for prothrombin binding.

DNA aptamers have a short half-life. So, RNA aptamers targeting thrombin have been developed. RNA modification increases the plasma stability. 2'-Fluoropyrimidine is an RNA aptamer that binds to thrombin. It inhibits the interaction of fibrinogen with thrombin and eventually causes anticoagulation. Another RNA aptamer is R9d14t. This 58 nucleotide long aptamer inhibits pro-exocite function.

REG1 is an anticoagulation system that includes RB006 and its oligonucleotide antidote RB007 (17-mer oligonucleotide). It is now in Phase-II clinical trial. RB006 is a 26 nucleotides long coagulation factor IXaspecific aptamer. This aptamer is conjugated to $40 \mathrm{kDa}$ PEG to reduce the renal filtration rate and capped with an inverted nucleotide at 3' terminus to reduce 3'exonuclease mediated degradation. RB006 binds to coagulation factor IXa and stops the coagulation process. RB007 is complementary to the 5' terminal region of RB006. RB007 disrupts the structure of RB006 and stops its anticoagulation (Keefe et al., 2010).

Aptamer in cancer treatment: AS1411 is a G-rich 26-mer oligodeoxynucleotide and present as a guaninequadruplex dimer in solution. AS1411 contains only guanines and thymines and is the first to enter clinical trials as a cancer therapeutic drug. AS1411 exhibits minimal toxicity in patients. AS1411 interacts with the protein nucleolin (Bates et al., 2009) (Figure 5). Nucleolin is involved in cell growth and happens to be overexpressed in many types of cancer such as lung cancer, breast cancer, prostate cancer etc. (Zhu et al., 2014). AS1411 inhibits cancer cell line through the following steps:

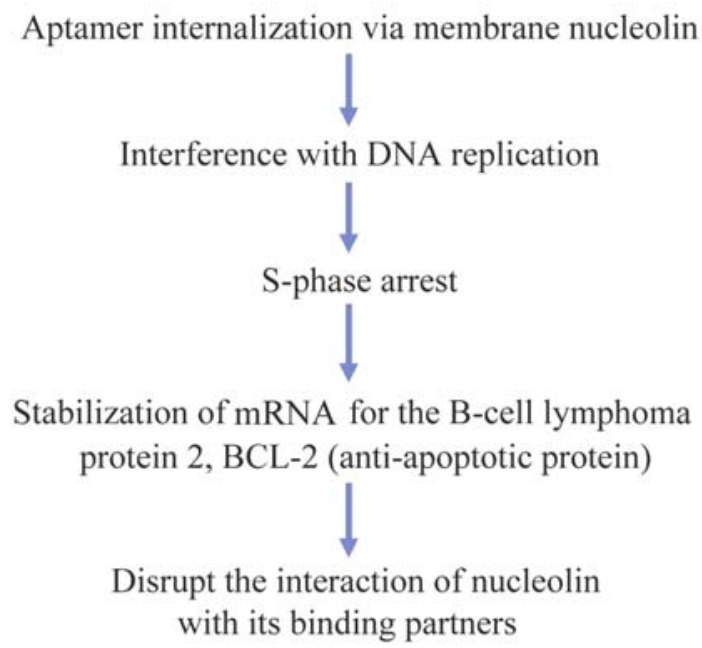

Figure 5. AS1411 in cancer treatment.

NOX-A12 is an RNA aptamer (Spiegelmer) that is 45 nucleotides long. NOX-A12 has reversed stereochemistry of the sugars and is linked to a $40 \mathrm{kDa}$ PEG at the 3' terminus. The mirror image of NOX-A12 makes the nucleotide resistant to endonuclease cleavage. Aptamer NOX-A12 binds with its targets CXCL12/SDF-1 (CXC chemokine ligand 12/stromal cell derived factor-1) (Ni et al., 2011) and make the cell susceptible to chemotherapeutic attack (Figure 6).

Delivery of cancer cell type specific aptamer: Targeted deliveries of aptamers are needed to improve their therapeutic efficacy and safety. After binding the aptamer with the targeted membrane protein, aptamers are internalized into the targeted cells where drugs are released and are free to exert their anticancer functions. For example, PLA (polylactide)-PEG or PLGA (polylactide-co-glycolide)-PEG nanoparticle encapsulated docetaxel conjugated with an RNA aptamer against the prostate specific membrane antigens (PSMA) was used to target prostate cancer cells (Farokhzad et al., 2004; Farokhzad et al., 2006).

Some aptamers conjugated directly with siRNA/mi-RNA deliver functional si-RNA/mi-RNA into cells. Prostate specific membrane antigen (PSMA) is overexpressed in prostate cancer cells and tumor vascular endothelium. The aptamer portion of the 
aptamersi-RNA chimera mediates binding to PSMA and si-RNA targets the expression of survival genes.
The chimera does not interact with the cells that do not express PSMA (McNamara et al., 2006).

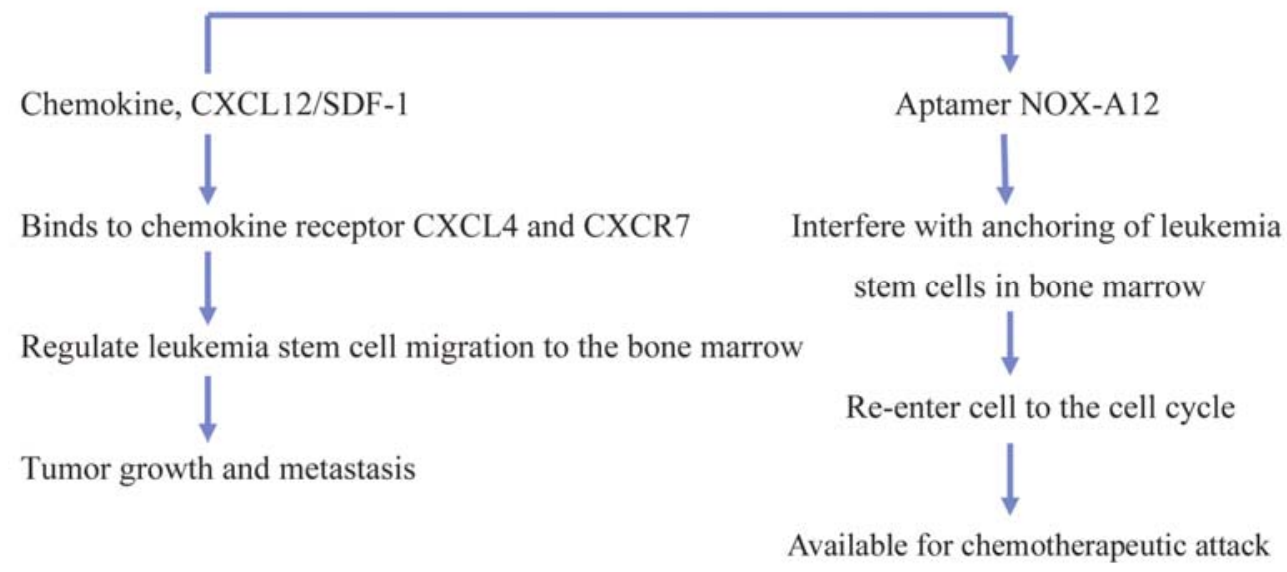

Figure 6. NOX-A12 for treatment of cancer.

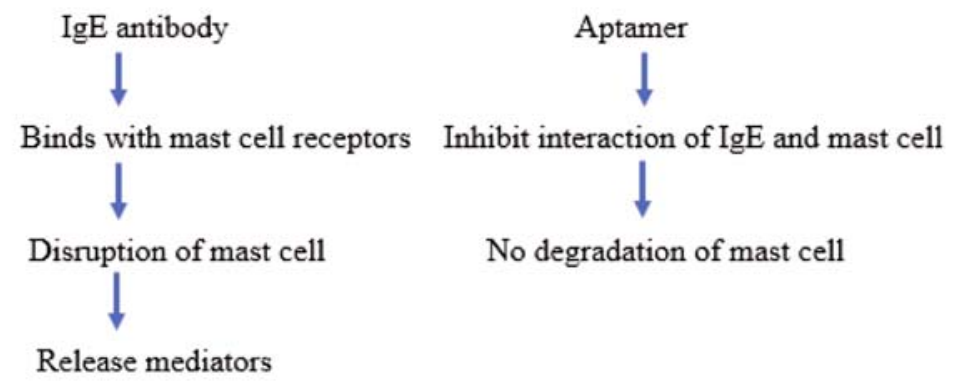

Figure 7. Aptamer against IgE.

Aptamers against autoantibodies: IgE is responsible for type one hypersensitivity reaction. Fc portion of the IgE binds to the receptor on mast cell and ruptures the cell membrane. Large amounts of mediators are released from the mast cells that are involved in hypersensitivity reaction (Figure 7). A DNA aptamer developed by Mendonsa group (Mendonsa et al., 2004) inhibits the interaction between antibody and mast cell and masks its effect.

Type II diabetic patients may produce insulin properly but their cells' insulin receptors may become inactive because of the presence of auto antibodies. A nuclease resistant RNA aptamer binds to murine insulin receptor antibody named MA20 that destroys the insulin receptor, thereby preventing diabetes (Le et al., 1996) (Figure 8). The similarity between murine and human insulin receptors has created the opportunity for RNA aptamers to reduce the symptoms of type II diabetes in humans as well.

Murine insulin receptor antibody MA20

RNA aptamer binds to MA20

MA20 can not destroy insulin receptor

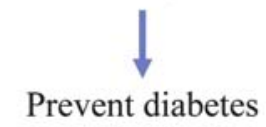

Figure 8. Diabetes treatment.

Kidney damage is the main complication of type II diabetes and it occurs due to the action of chemokine 
CCL2 (MCP-1). RNA aptamer NOX-E36 reduces the progression of kidney damage by targeting the chemokine CCL2 (Oberthur et al., 2015; Ninichuk et al., 2008; Maasch et al., 2008).

\section{Limitation of aptamers}

1. The half-life of unmodified aptamer is short. To increase the serum half-life, chemical modifications have been introduced into sugar moiety or phosphodiester linkage. 3' and/or 5', capping of nucleic acid strands protect aptamer from endonuclease attacks (Shigdar et al., 2013). Incorporation of inverted nucleotide at the 3 '-terminus decreases the serum endonuclease activity as 3' exonuclease activity in serum is much higher. Another method is the incorporation of fluoro or O-methyl group at the 2' position of the sugar moiety (Davydova et al., 2011). Incorporation of 2'amino pyrimidines, boranophosphate internucleotide linkages, 5modified pyrimidines, and/or 4'thio pyrimidines increases the nuclease resistance of aptamer (Shigdar et al., 2013).

2. Aptamers are usually small $(5-15 \mathrm{kDa})$ (Lakhin et al., 2013) so it is rapidly removed by renal filtration. Aptamer size can be increased through incorporation of bulky substances like PEG, cholesterol or peptides (Healy et al., 2004). Circulating half-life of $40 \mathrm{kDa}$ PEG-aptamer might be as long as one day (Burmeister et al., 2005). Another strategy is the cholesterol conjugation. Forty kilodalton PEG is conjugated with 2' fluoro/2'-O methyl modified aptamer so as to increase the serum half-life from 0.5 hours to 12 hours (Keefe et al., 2010).

\section{Conclusion}

Aptamers are a new emerging class of therapeutics which binds to its target with high affinity and specificity. They are susceptible to renal filtration and endonuclease cleavage. They can also be used for age related macular disease, autoimmune disease etc. Aptamer internalization occurs through receptormediated uptake, however, their processing, endosomal escape and drug release mechanisms are not completely understood. Toxicological aspects of aptamers also need to be investigated for clinical application. Cost effective aptamer production is still an important challenge.

\section{References}

Bates, P. J., Laber, D. A., Miller, D. M., Thomas, S. D. and Trent, J. O. 2009. Discovery and development of the Grich oligonucleotide AS1411 as a novel treatment for cancer. Exp. Mol. Pathol. 86, 151-164.

Bock, L. C. and Griffin, L. C. 1992. Selection of singlestranded DNA molecules that bind and inhibit human thrombin. Nature 355, 564.

Burmeister, P.E., Lewis, S.D. Silva, R.F., et al, 2005. Direct in vitro selection of a 2'-O-methyl aptamer to VEGF. Chem. Biol. 12, 25-33.

Davydova, A.S. Vorobjeva, M.A. and Venyaminova, A.G. 2011. Escort aptamers: new tools for the targeted delivery of therapeutics into cells. Acta. Naturae 3, 1229.

Farokhzad, O.C., Cheng, J. Teply, B.A., et al. 2006. Targeted nanoparticle-aptamer bioconjugates for cancer chemotherapy in vivo. Proc. Natl. Acad. Sci. USA. 103, 6315-6320.

Farokhzad, O.C., Jon, S., Khademhosseini, A., Tran, T.N., Lavan, D.A. and Langer, R. 2004. Nanoparticle-aptamer bioconjugates: a new approach for targeting prostate cancer cells. Cancer Res. 64, 7668-7672.

Griffin, L.C., Tidmarsh, G.F., Bock, L.C., Toole, J.J. and Leung, L.L. 1993. In vivo anticoagulant properties of a novel nucleotide-based thrombin inhibitor and demonstration of regional anticoagulation in extracorporeal circuits. Blood 81, 3271-3276.

Healy, J.M., Lewis, S.D., Kurz, M., et al. 2004. Pharmacokinetics and bio distribution of novel aptamer compositions. Pharm Res. 21, 2234-2246.

Keefe, A. D., Pai, S. and Ellington, A. 2010. Aptamers as therapeutics. Nature Rev. Drug Discov. 9, 537-550.

Kretz, C. A., Cuddy, K., Stafford, A. R., Fredenburgh, J. C., Roberts, R. and Weitz, J. I. 2010. HD1, a thrombin-and prothrombin-binding DNA aptamer, inhibits thrombin generation by attenuating prothrombin activation and thrombin feedback reactions. Thromb. Haemost. 103, 8393.

Kretz, C. A., Stafford, A. R., Fredenburgh, J. C. and Weitz, J. I. 2006. HD1, a thrombin-directed aptamer, binds exosite 1 on prothrombin with high affinity and inhibits its activation by prothrombinase. J. Biol. Chem. 281, 3747737485 . 
Lakhin, A.V., Tarantul, V.Z. and Gening, L.V. 2013. Aptamers: problems, solutions and prospects. Acta. Naturae 5, 34-43.

Lee, S.W. and Sullenger, B.A. 1996. Isolation of a nucleaseresistant decoy RNA that selectively blocks autoantibody binding to insulin receptors on human lymphocytes. $J$. Exp. Med. 184, 315-24

Maasch, C., Buchner, K., Eulberg, D., Vonhoff, S. and Klussmann, S. 2008. Physicochemical stability of NOXE36, a 40mer L-RNA (Spiegelmer) for therapeutic applications. Nucleic Acids Symp. Ser. (Oxf). 52, 61-62.

McNamara, J. O., Andrechek, E. R., Wang, Y., Viles, K. D., Rempel, R. E., Gilboa, E. and Giangrande, P. H. 2006. Cell type-specific delivery of siRNAs with aptamersiRNA chimeras. Nature Biotecnol. 24, 1005-1015.

Mendonsa, S.D. and Bowser, M.T. 2004. In vitro Evolution of functional DNA using capillary electrophoresis. J. Am. Chem. Soc. 126, 20-21.

Müller, J., Freitag, D., Mayer, G. and Pötzsch, B. 2008. Anticoagulant characteristics of HD1-22, a bivalent aptamer that specifically inhibits thrombin and prothrombinase. J. Thromb. Haemost. 6, 2105-2112.

Ni, X., Castanares, M., Mukherjee, A. and Lupold, S. E. 2011. Nucleic acid aptamers: clinical applications and promising new horizons. Curr. Med. Chem. 18, 42064214.

Ninichuk, V., Clauss, S., Kulkarni, O., Schmid, H., Segerer, S., Radomska, E., et al. 2008. Late onset of $\mathrm{CCL}_{2}$ blockade with the spiegelmer mNOXE36- 3'PEG prevents glomerulosclerosis and improves glomerular filtration rate in $\mathrm{db} / \mathrm{db}$ mice. Am. J. Pathol. 172, 628-37.
Oberthur, D., Achenbach, J., Gabdulkhakov, A., Buchner, K., Maasch, C., Falke, S., et al. 2015. Crystal structure of a mirror-image L-RNA aptamer (Spiegelmer) in complex with the natural L-protein target CCL2. Nat. Commun. 6, 6923.

Parashar, A. 2016. Aptamers in therapeutics. J.Clin. Diagnostic Rese. JCDR, 10, BE01.

Pendergrast, P. S., Marsh, H. N., Grate, D., Healy, J. M., and Stanton, M. 2005. Nucleic acid aptamers for target validation and therapeutic applications. J. Biomol. Techn. JBT, 16, 224.

Shigdar, S., Macdonald, J., O'Connor, M., Wang, T., Xiang, D., Al Shamaileh, H. and Kong, L. 2013. Aptamers as theranostic agents: modifications, serum stability and functionalization. Sensors, 13, 13624-13637.

Sundaram, P., Kurniawan, H., Byrne, M.E. and Wower, J. 2013. Therapeutic RNA aptamers in clinical trials. Eur. J. Pharm. Sci. 48, 259-71.

Tasset, D.M., Kubik, M.F. and Steiner, W. 1997. Oligonucleotide inhibitors of human thrombin that bind distinct epitopes. J. Mol. Biol. 272, 688-698.

Vinores, S.A. 2006. Pegaptanib in the treatment of wet, agerelated macular degeneration. Int. J. Nanomedicine. 1, 263.

Zhu, J., Huang, H., Dong, S., Ge, L. and Zhang, Y. 2014. Progress in aptamer-mediated drug delivery vehicles for cancer targeting and its implications in addressing chemotherapeutic challenges. Theranostics 4, 931-944. 